\title{
Dispositifs d'encadrement et d'évaluation du travail hospitalier : le rôle de la communication managériale pour la qualité
}

\section{Mouna El Gaïed}

\section{OpenEdition}

\section{Journals}

Édition électronique

URL : http://journals.openedition.org/communicationorganisation/1282

DOI : 10.4000/communicationorganisation. 1282

ISSN : 1775-3546

Éditeur

Presses universitaires de Bordeaux

Édition imprimée

Date de publication : 1 juin 2010

Pagination : $93-101$

ISBN : 978-2-86781-710-6

ISSN : 1168-5549

\section{Référence électronique}

Mouna El Gaïed, «Dispositifs d'encadrement et d'évaluation du travail hospitalier : le rôle de la communication managériale pour la qualité », Communication et organisation [En ligne], 37 | 2010, mis en ligne le 28 septembre 2011, consulté le 19 avril 2019. URL : http://journals.openedition.org/ communicationorganisation/1282 ; DOI : 10.4000/communicationorganisation.1282 


\title{
Dispositifs d'encadrement et d'évaluation du travail hospitalier : le rôle de la communication managériale pour la qualité
}

\author{
Mouna $\in \mid$ Goïed'
}

\section{Introduction}

Dans les milieux professionnels, la qualité s'apparente à des dispositifs managériaux issus de combinaisons assez complexes, mais néanmoins caractéristiques de discours et de stratégies opérationnelles qui agissent sur le travail et lui transmettent en même temps une charge symbolique positive. Nous saisissons ainsi et avant tout de la qualité sa capacité avérée à donner du sens au travail dans le but de valoriser l'image de l'organisation et obtenir l'adhésion des professionnels concernés.

Cette adhésion de la part du personnel est d'autant plus nécessaire que la mise en place des démarches qualité s'intègre à un processus d'aplatissement de la hiérarchie et à une volonté de faire participer les salariés, générant ainsi une plus grande responsabilisation de ces derniers. Ce n'est donc qu'à partir du moment où le professionnel s'approprie ces normes par un processus d'intériorisation que le travail produit va correspondre au travail prescrit.

Pour ce faire, les professionnels de l'encadrement mettent en place un «appareillage" complexe constitué de moyens de diffuser et de vérifier l'appropriation collective des discours sur la qualité comme le font les multiples formations au langage de la qualité (vocabulaire spécifique), aux audits internes (auto-évaluation) ou encore à la résolution de problèmes et dysfonctionnements dans une perspective d'amélioration continue de la qualité au travail. Ce qui revient à dire que l'élaboration de la «culture qualité » repose sur la création de nouvelles fonctions dans l'organisation relevant des domaines du management et de la communication.

01. Mouna El Gaied est maître de conférences en sciences de l'information et de la communication à Nancy 2 et membre du Centre de recherche sur les médiations de l'université Paul Verlaine de Metz. Elle est l'auteur de La transplantation managériale de I'hôpital paru en 2010 aux Éditions Universitaires Européennes. Mail: Mouna.El-Gaied@univ-nancy2.fr 
Si nous parlons ici de dispositifs managériaux en référence aux systèmes qualité, c'est pour mettre l'accent sur des modes d'organisation qui fonctionnent dans une double et même logique; entre action (faire et produire) et légitimation (faire croire et penser). En effet, le discours ne pourra marquer les esprits sans être relayé sur le terrain par un ajustement entre les activités technico-organisationnelles et la gestion symbolique des relations de travail. C'est en ce sens qu'un dispositif désigne l'agencement ou l'ajustement de deux formes d'existence; penser (savoir) et produire (pouvoir) qui s'interpénètrent de façon variable. Au-delà de ces affirmations, ce que nous cherchons ici à montrer c'est cette interdépendance du « matériel » et du «symbolique » car la pensée n'est pas seulement une pure illusion perceptive, mais correspond aussi à un processus objectif. Dans le cas des démarches qualité, nous pouvons assimiler les pratiques managériales à des dispositifs d'encadrement organisationnel et symbolique tels que nous venons de les décrire. Ainsi, il existe une intention rationnelle de mise en conformité concrète des nouvelles structures organisationnelles et des nouvelles formes de domination (contrôle et évaluation) avec leur incorporation pratique et symbolique par les salariés.

Par ailleurs, cet article sera pour nous l'occasion d'analyser le rôle de la communication managériale dans la mise en place et le maintien des nouveaux dispositifs qualité. Nous prenons ici l'exemple du champ hospitalier pour y avoir mené une étude empirique où la qualité apparaît comme vecteur de changement et de transformations organisationnelles et professionnelles. Mais de notre point de vue, il ne serait pas possible d'approcher la communication sans prendre connaissance de ce qui fait la particularité du champ qui nous intéresse du point de vue des valeurs et des codes de perception, et donc des représentations. Pour nous, parler de communication, c'est tout d'abord comprendre l'arrière-plan représentationnel et saisir cet univers symbolique en fonction duquel les messages prennent sens et peuvent ainsi circuler.

$\mathrm{Au}$ cours de notre analyse, nous allons dans un premier temps chercher à montrer les liens étroits mais néanmoins implicites, entre représentations sociales, communication managériale et démarches qualité pour revenir dans un deuxième temps sur le dispositif qualité à travers sa double dimension à savoir l'encadrement et l'évaluation.

\section{La communication managériale pour la qualité, facteur de transformations organisationnelles}

Les démarches qualité constituent un moment où des transformations s'opèrent dans la manière de concevoir et de réaliser le travail et l'organisation. Compte tenu de cela, la communication managériale a pour fonction de rendre légitimes ces transformations en agissant sur les représentations sociales sous-jacentes en vue de les rendre plus adéquates avec les objectifs managériaux. À partir de là, le sens attribué aux transformations du travail et de l'organisation se trouve au centre des préoccupations de la 
communication et du management. Ce changement des représentations qui accompagne impérativement le changement des structures et des modes de fonctionnement peut ainsi être considéré comme au moins tout autant important que ces derniers.

Ainsi, plusieurs auteurs ont cherché à établir le lien entre la communication et la construction des représentations. La communication n'est pas un phénomène neutre en ce sens qu'elle implique des processus d'influence et nécessite ainsi d'être élaborée pour atteindre ses objectifs. La communication apparaît dès lors comme un vecteur de transmission du langage, lui-même porteur de représentations. Cette fonction symbolique de la communication soutiendrait toutes les actions de communication, mais se laisse en même temps véhiculer par elles. Autrement dit, le symbolique n'est concrétisé qu’à partir du moment où il est véhiculé par le langage et autres signes à travers des actions de l'ordre du fonctionnel.

Pour les démarches qualité, cette fonction fédératrice du discours est plus que jamais d'actualité, dans le sens où la qualité constitue désormais l'une des pièces maîtresses de l'argumentaire, visiblement irréfutable, mise en avant par le management. Frederik Mispelblom a développé une analyse sur le rôle de la communication dans la construction des représentations (Mispelblom, 2000). L'auteur part d'une étude sur l'introduction de l'assurance qualité dans une entreprise. Les démarches qualité avec la mise en place des écrits formalisés: notes de services, procédures, directives, méthodes écrites et normes visent à construire une représentation unifiée de l'entreprise. Les procédures écrites et les nouveaux moyens de contrôle ont une emprise sur les représentations qui gouvernent les salariés dans leur travail au quotidien. L'auteur ajoute que la définition des activités contenues dans ces écrits est rattachée à la direction de l'entreprise. Cette description des activités et des postes correspond selon lui « moins à la description de la réalité du travail, mais ce qu'il devrait être aux yeux de ceux qui le contrôlent » (Mispelblom, 2000, p. 220).

Cette fonction de construction des représentations se retrouve également développée par Bernard Floris. L'auteur analyse la capacité de symboliser ou de signifier « qui consiste à percevoir et à produire des représentations subjectives de la réalité » (Floris, 2000, p. 175). Et il estime que l' « ingénierie symbolique » est l'activité rationalisée qui vise à produire et à diffuser des représentations conformes aux objectifs du management. Pour lui, la communication serait une des formes actuelles de production et de diffusion des processus symboliques dans les rapports de l'entreprise avec les autres institutions sociales et dans les relations de leurs directions avec les salariés. Cette fonction regroupe tous les actes de production et d'attribution de sens aux objets, aux institutions et aux individus en rapport avec l'entreprise.

Il est ainsi apparu durant les années 1980 que des nouveaux « dispositifs d'encadrement organisationnel et symbolique de travail agissent sur 
les catégories de perception, de représentation et d'action des salariés " (Floris, 2000, p. 182). Le "management symbolique" (Bourdin, 2000, p. 252) a pour objectif d'agir sur les représentations des salariés pour arriver à une perception unifiée de l'organisation, et à la production d'une «vision consensuelle des rapports de travail» (De Crescenzo, Floris, 2000, p. 166). De ce fait, la communication managériale construit un modèle de comportement qui s'appuie sur la domination symbolique.

C'est aussi cela que Frederik Mispelblom cherche à mettre en évidence en parlant de l' «entreprise politique ». Une question qui se pose avec la mise en place et le développement de la qualité dans le monde du travail. En ce sens que les démarches qualité, proprement dites, s'inscrivent à leur tour dans une longue tradition politique ayant conduit, dans un premier temps, les gouvernements à se préoccuper du bonheur de leurs sujets devenus citoyens (Mispelblom, 1999).

Ceci est d'autant plus vrai que le thème de la qualité montre bien que « les entreprises exercent des fonctions de plus en plus explicitement politiques, dont certaines étaient auparavant réservées à l'appareil d'État: formation et éducation de leur personnel, mais aussi organisation et éducation de leurs clients. Le thème de l' " entreprise citoyenne » en témoigne, une partie des dirigeants d'entreprise se reconnaissent des responsabilités à l'égard de toute la société, du chômage, des exclus et de la "fracture sociale ", bref de la cohésion sociale et du consensus » (Mispelblom, 1999, p. 265).

Ce sont donc aujourd'hui les entreprises elles-mêmes qui, par le biais de la qualité, veulent le bien des citoyens ce qui est, à notre avis, assez révélateur de la façon la plus efficace pour réhabiliter l'image de l'entreprise capitaliste et réaliser sa réconciliation avec la société. Comme le souligne Bernard Floris, l'entreprise privée étant devenue «le modèle d'identification sociale et de légitimation institutionnelle »(Floris, 1996), elle s'est laissée importer dans le secteur public et l'hôpital ne semble pas avoir échappé à cette tendance comme le précisait déjà J.-P Le Goff en 1995 (Le Goff, 1995).

\section{L'encadrement et l'évaluation, les maîtres mots du management par la qualité}

C'est en partant d'une acception foucaldienne (Foucault, 1975) que le management pourrait être défini comme une «technologie politique»; il s'agit d'un dispositif organisateur de discours qui guident des actes, d'un savoir inscrit dans des techniques verbales et mentales qui produit du pouvoir. Il apparaît ainsi que derrière l'idéal de la qualité, se cache un dispositif de rationalisation de l'organisation du travail allant de pair avec une transparence et un contrôle généralisés. La qualité aurait en effet pour corollaire la disparition de toute opacité dans la production, pour les salariés comme pour un « regard externe ». Elle correspondrait au déploiement d'une conception très idéalisée de la communication qui exclurait tout conflit et tout dysfonctionnement (Olivesi, 2002). 


\section{Le rôle de l'encadrement}

Le management et la qualité sont souvent perçus comme les deux facettes d'une même réalité. Hans Ulrich définit le management comme « un ensemble d'activités nécessaires à la conception, au contrôle et au développement de systèmes sociaux déterminés » (Ulrich, 1989). Ces activités sous-entendent, pour les responsables d'établissements, la capacité de concevoir une représentation du système dont ils font partie afin de pouvoir y agir (y mettre la main, maneggiare) (Ducalet, Laforcade, 2000).

Lacception contemporaine du management concerne tout autant la dimension stratégique que celles de l'animation et de la gestion des ressources humaines (Ducalet, Laforcade, 2000). La qualité qui cherche fondamentalement à s'imposer parmi les différents changements auxquels l'organisation est confrontée, a nécessairement besoin de légitimation. Elle fait, pour y parvenir, appel au management comme moyen de gérer le social et susciter son adhésion.

De là, nous nous rendons compte que ce système qualité reposerait sur une gestion des subjectivités cherchant à mobiliser à l'intérieur de l'hôpital les professionnels autour des objectifs des décideurs en matière de mise en conformité des pratiques, de l'organisation et enfin des modes de pensée. Dans le cadre des formations à la qualité par exemple ou encore des Systèmes Qualité des Services (l'équivalent des cellules qualité), les personnes qui y participent se voient confrontées à ce type de management dans la mesure où l'on cherche à provoquer chez eux l'adhésion aux changements occasionnés par la mise en place du système dans l'établissement. Ce management comprendrait ainsi une fonction de l'ordre du symbolique.

À ce niveau de la réflexion, il semblerait nécessaire de rappeler les liens, plus au moins implicites, entre ce type de management, la communication faite en direction du personnel hospitaliers et enfin la démarche qualité. Cette dernière, en provoquant des changements de fond par rapport au travail et au sens que les acteurs lui attribuent, aurait besoin de faire appel puis de développer des fonctions de gestion des ressources humaines. Ces mêmes fonctions auraient, à leur tour, la mission de modifier les représentations subjectives pour mieux faire accepter le changement, mais aussi faire asseoir une nouvelle conception de ce monde du travail. La mission des « réaménagements symboliques » est assurée par la communication institutionnelle au sens large du terme. Que faut-il donc entendre par gestion ou ingénierie symbolique dont les fonctions sont désormais confiées à des services spécialisés (services qualité et évaluation, etc.)? Sans doute, la capacité de produire et de diffuser des représentations subjectives de la réalité. Celles-ci établissent un lien conventionnel permanent entre des phénomènes physiques sociaux ou mentaux et des significations (symboles ou signes). La fonction symbolique de la communication fait appel à un ensemble d'attributions de sens aussi bien aux objets qu'aux institutions et aux individus qui les intègrent. Ce management peut donc être défini, en 
faisant référence à Michel Foucault, comme une « technologie politique » qui permet « d'imposer une tâche ou une conduite à une multiplicité d'individus » (Foucault, 1975, p. 240). Par là, le philosophe cherche à démontrer que les régimes de pouvoir sont toujours indissociablement des régimes de savoir. En effet, un dispositif désigne l'agencement ou l'ajustement de deux formes qui s'interpénètrent de façon variable.

Un dispositif d'encadrement organisationnel et symbolique exerce un double travail symbolique sur les catégories de perception, de représentation et d'action des salariés. D'une part directement par une action sur les représentations et le langage au niveau d'une gestion symbolique autonomisée; et d'autre part, indirectement par l'articulation concrète entre ces catégories de perception et les pratiques professionnelles. La qualité elle-même s'appuie sur des procédures écrites et autres productions matérielles allant jusqu'à transformer le contenu du travail et son organisation. Dans le cas des structures hospitalières, nous pouvons assimiler les formations qualité, ainsi que les groupes de travail autour de l'objectif de la qualité à des dispositifs d'encadrement organisationnel et symbolique. Cet encadrement est d'autant plus nécessaire qu'il opère sur une base de confrontation de logiques différentes, voire contradictoires, à savoir les logiques professionnelles antérieures d'une part et celle de la qualité d'autre part. Ce qui revient à dire que la communication et le management à l'hôpital se doivent d'intervenir afin d'accomplir un changement dans les mentalités et dans les façons de faire au quotidien pour pouvoir faire accepter les procédures, notamment en ce qui concerne la généralisation de l'écriture comme outil de référence au travail. La légitimation apparaît ainsi comme une justification laissant entrevoir une difficulté à instaurer au sein de l'hôpital la nouvelle doctrine managériale. Un tel changement semble requérir des justifications que la communication et le management prennent en charge.

\section{Qualité et évaluation du travail hospitalier}

L'évaluation est au cœur des démarches qualité. Elle constitue, en effet, le moment où l'on vient mesurer le degré de correspondance entre le travail conçu, autrement dit, reconnu de qualité et décrit dans les procédures, et le travail réalisé par les professionnels. Avec l'accréditation, ayant pour objectif la validation des actions menées par l'établissement de santé et par ses personnels dans le sens d'une mise en conformité des pratiques, l'évaluation (l'auto-évaluation dans un premier temps et l'évaluation dans un second temps par les experts visiteurs de la HAS : Haute Autorité de Santé) vient justement témoigner du degré de correspondance entre les référentiels et le travail fourni. Mais, l'accréditation et la démarche qualité présentent une certaine différence et ne peuvent donc pas être totalement confondues, bien qu'elles soient très liées dans la réalité. Pour mieux expliciter leur principale différence, nous pourrions dire que l'accréditation est le versant officiel des démarches qualité 
menées dans les établissements de santé, tout comme la certification ISO selon les critères de l'Organisation Internationale de Normalisation qui, pour sa part, atteste d'un niveau de qualité reconnu des produits ou des services de l'entreprise en question.

Une des idées phares autour desquelles est élaborée la communication qualité à l'hôpital rejoint justement cette distinction entre procédure d'accréditation d'une part et démarche d'amélioration de la qualité d'autre part. Vue sous cet angle, l'accréditation apparaît comme un moment important dans le " parcours de qualité » d'une organisation. Pour les managers, elle n'est qu'un des moyens d'atteindre la démarche qualité en rendant possible un constat objectif qui va à son tour aider l'encadrement à remédier aux dysfonctionnements repérés. À y voir de plus près, la fonction évaluative rejoint les procédures et l'usage qui en est fait. En effet, le résultat des évaluations s'accompagne généralement de sanctions pour l'établissement concerné: mises à niveau et actions correctives, obtention ou refus de l'accréditation, etc. Ces évaluations peuvent avoir des retentissements importants sur les représentations de la qualité par les salariés, car elles peuvent être perçues comme de véritables sanctions.

Ainsi l'introduction des méthodes quantitatives dans la mesure de l'activité, notamment du corps médical, introduit la « culture de la mesure » dont parle Valérie Carayol (Carayol, 2000), jusque-là limitée à la production, dans tous les secteurs de l'activité sociale et sanitaire. À partir de là, le travail des médecins, considéré comme une suite de processus, fait à son tour, l'objet de contrôles et d'évaluations. D'un autre côté, Pierre Delcambre (Delcambre, 1997) insiste, parmi les fonctions qu'on attribue généralement à l'écriture, sur son rôle de « mémoire artificielle ». En ce sens, « inscrire » revient à laisser des traces et donc permettre aux autres d'aller au-delà de l'expérience individuelle.

Dans le champ médical, la demande qu'une trace soit conservée et l'imposition d'un travail de trace génèrent bien des réticences face à cet exercice considéré comme point de départ à l'évaluation et au contrôle. Les médecins sont, en effet, très autonomes dans un double sens; ils n'acceptent pas l'éventualité d'un contrôle de leur exercice (la trace) et ont du mal à se soumettre à une source extérieure, notamment aux normes de la qualité. D'un autre côté et parmi les implications de l'acte de l'écriture, certains médecins évoquent l'antagonisme entre les exigences de la qualité et le « secret médical ». À partir de là, l'attachement des médecins à la confidentialité des données et au secret médical est ainsi avancé comme argument pour s'opposer à la « qualité ». Toutefois, quelles qu'en soient les modalités, ce passage à l'écrit, dans le cadre de l'application des démarches qualité, induit un changement de régulation du travail que la plupart des médecins n'approuvent pas car, d'une certaine façon, il les dépossède de leurs savoirs informels et les soumet à une source extérieure du travail. Ainsi, en mettant à jour les zones de liberté des médecins, il entraîne leur perte d'autonomie. Ce qui revient à constater que cette formalisation des savoirs recèle l'ambiguïté consistant à transformer des 
règles autonomes en règles hétéronomes qui serviront ensuite au contrôle (Laurens, 2000). On peut également y voir le développement d'une logique de pouvoir qui cherche à s'imposer de façon librement consentie (Domenc, Marquié, 1999).

\section{Conclusion}

Pour les structures hospitalières, l'avènement d'un nouveau modèle relationnel et de nouveaux thèmes d'identification au travail pose le problème de leur compatibilité avec les modèles préexistants. En d'autres mots, il est important d'évoquer les risques d'incompatibilité et de " rejet » de l'univers symbolique naissant à partir du moment où tous les secteurs de l'ordre institutionnel sont depuis longtemps bien intégrés dans un cadre de référence qui constitue un univers au sens littéral du terme, dans la mesure où toute expérience humaine relevant de ce domaine peut être conçue comme prenant place en son sein (Berger, Luckmann, 1996). Ce qui revient à dire qu'il existe toujours des risques de rejet de ces nouveaux modèles relationnels. En ce sens, les sujets concernés par un tel changement, peuvent vouloir maintenir leurs univers symboliques préexistants. Dans l'état actuel des choses, la confrontation des logiques professionnelles au niveau des organisations hospitalières semble se jouer en faveur des identités professionnelles antérieures car il s'agit de valeurs si ancrées dans le champ professionnel en question, à savoir le champ hospitalier, et préalablement intériorisées par une grande majorité des soignants, particulièrement les moins jeunes parmi eux. Ceci dit, l'exemple du système de santé américain, si proche de la logique du privé, nous laisse penser que d'autres modèles et conceptions de la santé pourraient arriver un jour à s'imposer comme évidence. La communication aura ainsi comme rôle de contribuer à légitimer ces nouvelles visions afin d'y faire adhérer les personnels.

\section{BIBLIOGRAPHIE}

BERGER Peter, LUCKMANN Thomas. La construction sociale de la réalité, Paris: Armand Colin, 1996, 357 p.

BOURDIN Sylvie, «Pour une médiologie des organisations ». In: DELCAMBRE P., Coord. Communications organisationnelles, objet, pratiques, dispositifs, Rennes: PUR, 2000, pp. 243-259.

CARAYOL Valérie, Pour une approche communicationnelle de la qualité, Communication E organisation, $2^{\mathrm{e}}$ semestre 2000, n 17 , pp. 239-255.

DE CRESCENZO Jean-Claude, FLORIS Bernard. « La gestion participative comme objet de communication organisationnelle ». In:DELCAMBRE P., Coord. Communications organisationnelles, objet, pratiques, dispositifs, Rennes: PUR, 2000, pp. 159-174.

DELCAMBRE Pierre. Écriture et communications de travail, France: Presses Universitaires du Septentrion, 1997, $325 \mathrm{p}$. 
DOMENC Michel., MARQUIE Hélène. «Les acteurs dans les démarches qualité: soumission ou coproduction?", Sciences de la société, Presses Universitaires du Mirail, février 2000, n 46, pp. 35-48.

DUCLAET Philippe., LAFORCADE Michel. Penser la qualité dans les institutions sanitaires et sociales, S. Arslan, 2000, 320 p.

FLORIS Bernard. La communication managériale. La modernisation symbolique des entreprises, Grenoble: PUG, 1996, 272 p.

FLORIS Bernard. La gestion symbolique entre ingénierie et manipulation, Sciences de la société, Presses Universitaires du Mirail, octobre 2000, nº 50/51, pp. 173-196.

FOUCAULT Michel. Surveiller et punir, Paris : Gallimard, 1975, 318 p.

LAURENS Patrick. Qualité et organisation. La preuve par la communication, Sciences de la société, Presses Universitaires du Mirail, octobre 2000, n 50/51, pp. 241-260.

LE GOFF Jean-Pierre. Le mythe de l'entreprise, Paris: La Découverte, 1995, 307 p.

MISPELBLOM BEYER Frederik. Au-delà de la qualité. Démarches qualité, conditions de travail et politiques du bonheur, Syros-La Découverte, 1999, 305 p.

MISPELBLOM BEYER Frederik. Communication organisationnelle et parlers ordinaires. Les dimensions politiques du langage, Sciences de la société, Presses Universitaires du Mirail, octobre 2000, n 50/51, pp. 217-240.

OLIVESI Stéphane. La communication au travail, Grenoble: PUG, 2002, 164 p.

ULRICH Hans. Pensée globale et management: résoudre les problèmes complexes, Éditions d'Organisation, 1989, 314 p.

Résumé : Les dispositifs d'encadrement et d'évaluation au travail tels que les démarches qualité exercent une double action, d'une part sur les représentations et le langage au niveau d'une gestion symbolique autonomisée ; et d'autre part, par l'articulation concrète entre ces catégories de perception et les pratiques professionnelles. Dans le cas des démarches qualité à l'hôpital, le management s'assimile à une intention rationnelle de mise en conformité des nouvelles structures organisationnelles avec leur incorporation pratique et symbolique par les professionnels.

Mots-clés : dispositifs, management, qualité, communication, hôpital, encadrement, évaluation.

Abstract : The implements of supervision and evaluation at work such as total quality control exert a double action, on one hand on presentations and language at the level of autonomous symbolic management ; and on the other hand, by a concrete articulation between these perception categories and professional practices. In the case of total quality control at the hospital, management is comparable to a rational intention of compliance of new organizational structures with their practical and symbolic incorporation by the professionals.

Keywords : implements, management, quality, communication, hospital, supervision, evaluation 
